\title{
The scuttle fly (Diptera: Phoridae) assemblages of a wildfire- affected hemiboreal old-growth forest in Tyresta (Sweden)
}

\author{
Ewa Durska, James Bonet \& Bert Viklund
}

\begin{abstract}
Durska, E., Bonet, J. \& Viklund, B. 2010: The scuttle fly (Diptera: Phoridae) assemblages of a wildfire-affected hemiboreal old-growth forest in Tyresta (Sweden). — Entomol. Fennica 21: 19-32.
\end{abstract}

In natural forests, fire is an important disturbance factor and many studies have been carried out concerning its effect on different ecosystems, but no studies have previously been done considering the scuttle flies in hemiboreal forests. Here, we carried out an ecological investigation of the scuttle fly assemblage in a hemiboreal old-growth forest in Tyresta National Park and Nature Reserve (Sweden) from material collected, using Malaise traps, after wildfires in 1997 and 1999. We evaluated abundances of species, dominance structure, species richness (by non-parametric species richness method - Chao 1) as well as phenology. The most abundant species of the dominant group (i.e. Megaselia pleuralis, M. nigriceps, M. pulicaria-complex and $M$. brevicostalis) are multivoltine and saprophagous, displaying spring and late summer/autumn activity.

E. Durska, Museum and Institute of Zoology, PAS, Department of Ecology and BiodiversityWilcza 64,00-679Warsaw,Poland; E-mail:edurska@miiz.waw.pl J. Bonet, Swedish Museum of Natural History, Department of Entomology P.O. Box 50007, SE-10405 Stockholm, Sweden; E-mail: james.bonet@nrm.se

B. Viklund, Swedish Museum of Natural History, Department of Entomology P.O. Box 50007, SE-10405 Stockholm, Sweden; E-mail: bert.viklund@nrm.se

Received 8 April 2009, accepted 8 June 2009

\section{Introduction}

Fire represents a prevalent natural factor in the dynamics of boreal and hemiboreal forest ecosystems. The influence of fire on the insect fauna is both short term (direct) and long term through the influence of the subsequent vegetational succession (Holliday 1991). The number and size of wildfires, as well as the "fire-free" periods connected with weather conditions, have important ecological consequences by enhancing local and regional heterogeneity and may be especially important for organisms that colonize recently burnt areas through migration (Danks \& Foottit 1989,
Koivula et al. 2006, Niklasson \& Granström 2000, Southwood 1962, Zackrisson 1977).

In natural forests, fire is an important disturbance factor and many studies have been performed on their effects on different ecosystems (DeBano et al. 1998). Wildfire effects on invertebrates have been considered numerous times (Swengel 2001, Wikars \& Schimmel 2001, Moretti et al. 2002). Wildfires have been shown to increase the species richness of insects, especially among beetles and butterflies (Muona \& Rutanen 1994, Toivanen \& Kotiaho 2007, Hirowatari et al. 2007).

Phoridae (scuttle flies) is one of the most 


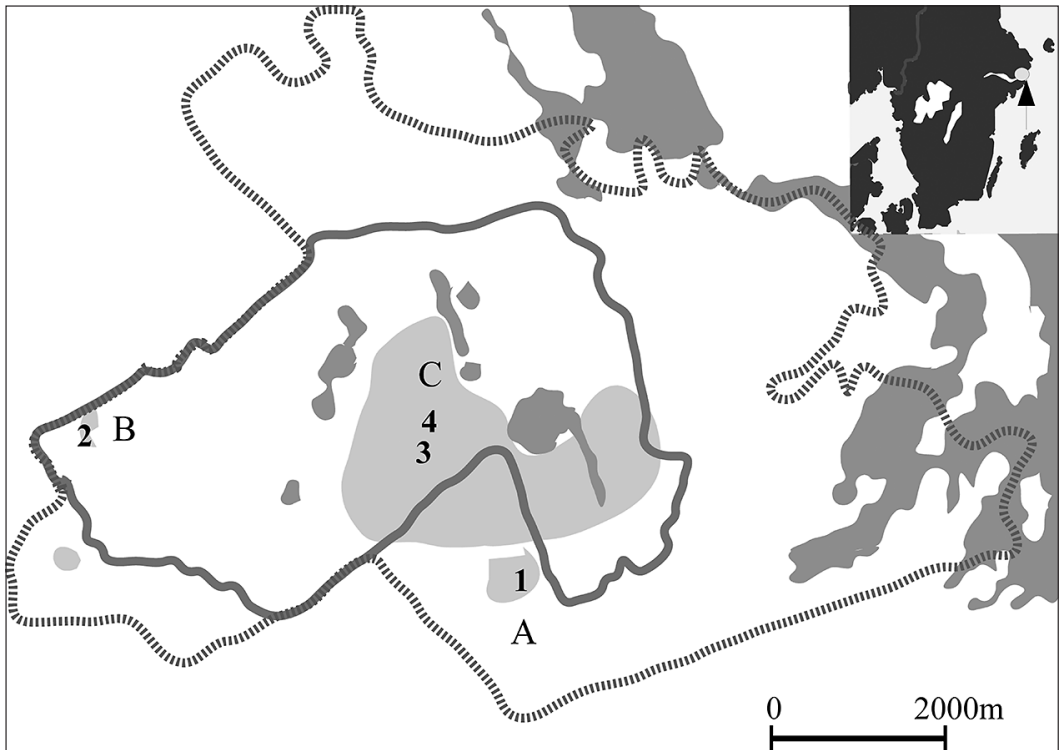

Fig. 1. Location of the Tyresta National Park (continuous line) and the Nature Reserve (dashed line) study area. Dark grey areas indicate lakes and light grey areas wildfire affected forest areas. The black arrow shows the position in Sweden and proximity to Stockholm (white circle). Numbers $(1,2,3,4)$ refer to the position of the traps and letters $(A, B, C)$ to wildfire sites. abundant and diverse Diptera families in the majority of terrestrial ecosystems (Disney 1994). Scuttle flies also constitute a major part of the pioneer fauna recolonizing habitats after disturbances such as clear cuttings (Durska 2001, 2006, 2009). Prescher et al. (2002) studied the scuttle fly assemblages in a chestnut forest in Switzerland after wildfires.

However, no studies have previously been done considering wildfire effects on the scuttle flies in hemiboreal forests (i.e. in the northern part of the temperate zone where the deciduous forest mixes with the taiga).

An extensive arthropod survey that had been running for four years (1999-2002) in a Swedish old-growth forest in the Tyresta National Park and Nature Reserve provided us a unique opportunity to examine the effect of wildfires on the scuttle fly assemblage structure and to improve our knowledge of Phoridae diversity in Sweden. The raw data was problematic since the survey originally was not intended for advanced statistical analyses, i.e., the samples were not exactly from the same locations within the area together with differences in periods and trap days (sampling effort).

As the observed number of species and individuals increase with increased sampling effort the samples may not be comparable i.e., it will likely result in differences in diversity indi- ces/species richness and may only reflect sampling effort (Scharff et al. 2003, Colwell \& Coddington 1994). Thus, standardization of the number of individuals and species among sites may improve our understanding of abundance patterns. Non-parametric species richness estimation methods have been used to control for unequal sampling effort (Walther \& Martin 2001). The purpose of this paper is to document and consider the abundance, dominance structure and phenology of Phoridae in the Tyresta area near Stockholm.

\section{Material and methods}

\subsection{Material}

The material studied was collected in 1999 in Tyresta National Park and Nature Reserve (Tyresta Forest), which is situated 30 kilometers south of Stockholm, Sweden $\left(59^{\circ} 9^{\prime}-59^{\circ} 13^{\prime} \mathrm{N}\right.$, $18^{\circ} 12^{\prime}-18^{\circ} 24^{\prime}$ E) (Fig. 1). Scuttle flies were collected using Malaise traps (Marris House Nets $^{\mathrm{TM}}$ ), with collecting heads containing 50\% propylene glycol and a few drops of detergent to facilitate submersion of trapped insects. Identifications were done under a dissecting microscope with the material transferred to glycerol. Unidentified Megaselia material was set aside as Mega- 
Table 1. List of species (males) with number of individuals ( $n$ ) given for 1999, together with the number of trap days for each sample. Relative abundance (\%) is given for the total sample. Dominant species for the equal sampling effort with dominance $\geq 1 \%$ (see Table 2) are shown in bold type. Species with * are new to Sweden, $n$ $=$ number of individuals, $T . S=$ total sample.

\begin{tabular}{lrrrrrrrrrr}
\hline Sample & 29 & 27 & 9 & 10 & 17 & 19 & 18 & 20 & T.S & T.S \\
\hline Trap days & 24 & 49 & $\begin{array}{r}37 \\
n\end{array}$ & $\begin{array}{r}44 \\
n\end{array}$ & $\begin{array}{r}28 \\
n\end{array}$ & $\begin{array}{r}28 \\
n\end{array}$ & $\begin{array}{r}42 \\
n\end{array}$ & $\begin{array}{r}42 \\
n\end{array}$ & $\begin{array}{r}294 \\
n\end{array}$ & $\begin{array}{r}294 \\
\%\end{array}$ \\
\hline
\end{tabular}

Anevrina unispinosa (Zetterstedt, 1860)

*Beckerina umbrimargo (Becker, 1901)

Conicera floricola Schmitz, 1938

Conicera similis (Haliday, 1833)

Conicera tibialis Schmitz, 1925

Diplonevra nitidula (Meigen, 1830)

Hypocerides nearcticus (Borgmeier, 1966) Megaselia abdita Schmitz, 1959

M. aculeata (Schmitz, 1919)

M. aequalis (Wood, 1909)

M. affinis (Wood, 1909)

M. albicaudata (Wood, 1910)

M. albiclava Schmitz, 1926

M. albocingulata (Strobl, 1906)

M. annulipes (Schmitz, 1921)

M. barbulata (Wood, 1909)

M. basispinata (Lundbeck, 1920)

M. berndseni (Schmitz, 1919)

M. bifida Disney, 1983

M. brevicostalis (Wood, 1910)

M. campestris (Wood, 1908)

M. ciliata (Zetterstedt, 1848)

M. communiformis (Schmitz, 1918)

M. conformis (Wood, 1909)

M. consetigera (Schmitz, 1925)

M. crassipes (Wood, 1909)

M. curvicapilla Schmitz, 1947

M. differens Schmitz, 1948

M. discreta (Wood, 1909)

M. diversa (Wood, 1909)

M. drakei Disney, 1984

M. eccoptomera Schmitz, 1927

M. elongata (Wood, 1914)

M. emarginata (Wood,1908)

M. excavata Schmitz, 1927

M. flavescens (Wood, 1909)

M. flavicans Schmitz, 1935

M. frameata (Schmitz, 1927)

M. fungivora (Wood, 1909)

M. furva Schmitz, 1929

M. fuscipalpis (Lundbeck, 1920)

M. fuscoides Schmitz, 1934

M. fuscovariana Schmitz, 1933

M. gartensis Disney, 1985

M. giraudii-complex

M. glabrifrons (Wood, 1909)

M. gregaria (Wood, 1910)

${ }^{*}$ M. henrydisneyi Durska, 1998

M. hibernans Schmitz, 1934

$\begin{array}{rrrrrrrrrr}- & 2 & - & - & - & - & - & - & 2 & 0.03 \\ - & 1 & - & - & - & - & - & - & 1 & 0.02 \\ - & - & 2 & - & - & - & - & - & 2 & 0.03 \\ - & - & 3 & - & - & - & - & - & 3 & 0.05 \\ - & - & 2 & - & - & - & - & - & 2 & 0.03 \\ - & - & 1 & - & 18 & - & - & 2 & 21 & 0.32 \\ - & - & - & - & 3 & - & - & - & 3 & 0.05 \\ - & - & 1 & - & - & - & - & - & 1 & 0.02 \\ - & - & - & - & - & - & 1 & - & 1 & 0.02 \\ - & 1 & - & - & - & - & - & - & 1 & 0.02 \\ - & 1 & 5 & 5 & - & - & - & - & 11 & 0.17 \\ - & 5 & - & - & - & - & - & - & 5 & 0.08 \\ - & 3 & - & - & - & - & - & - & 3 & 0.05 \\ - & 10 & 5 & 3 & 4 & 20 & 1 & 1 & 44 & 0.67 \\ - & 1 & - & - & - & - & - & - & 1 & 0.02 \\ - & - & - & - & - & 1 & - & - & 1 & 0.02 \\ - & 14 & 19 & 2 & 3 & 9 & - & - & 47 & 0.72 \\ - & 2 & 4 & - & 1 & - & - & - & 7 & 0.11 \\ - & 1 & - & - & 1 & 1 & - & - & 3 & 0.05 \\ - & 125 & 185 & \mathbf{1 7} & \mathbf{4 8} & \mathbf{6 5} & - & \mathbf{8} & 448 & 6.83 \\ - & - & - & 1 & - & - & - & - & 1 & 0.02 \\ - & 3 & - & - & - & - & - & - & 3 & 0.05 \\ - & 2 & - & - & - & - & - & - & 2 & 0.03 \\ 2 & 1 & - & - & - & - & - & - & 3 & 0.05 \\ - & 13 & - & - & - & - & - & - & 13 & 0.20 \\ - & 1 & - & - & - & - & - & - & 1 & 0.02 \\ 3 & 27 & 1 & - & - & - & - & - & 31 & 0.47 \\ - & 6 & - & - & - & - & - & - & 6 & 0.09 \\ - & 5 & - & 1 & 4 & 1 & - & - & 11 & 0.17 \\ - & 18 & - & - & \mathbf{9} & \mathbf{1 9} & \mathbf{7} & \mathbf{1 8} & \mathbf{7 1} & 1.08 \\ - & 6 & - & 2 & - & - & - & - & 8 & 0.12 \\ - & 2 & - & - & - & - & - & - & 2 & 0.03 \\ - & - & 1 & - & - & - & - & - & 1 & 0.02 \\ - & 20 & - & 1 & - & - & - & - & 21 & 0.32 \\ - & - & - & 5 & - & 7 & 5 & 19 & 36 & 0.55 \\ - & 1 & - & - & - & - & - & - & 1 & 0.02 \\ 2 & 2 & - & - & - & - & - & - & 4 & 0.06 \\ - & 8 & 1 & - & - & 1 & - & 1 & 11 & 0.17 \\ - & 58 & - & - & - & - & - & 1 & 59 & 0.90 \\ - & 1 & - & 1 & - & 2 & - & 4 & 8 & 0.12 \\ - & 3 & - & - & - & - & - & - & 3 & 0.05 \\ - & 1 & - & - & - & - & - & - & 1 & 0.02 \\ - & 1 & - & - & - & - & - & - & 1 & 0.02 \\ - & 1 & - & - & - & - & - & - & 1 & 0.02 \\ - & 1 & - & - & \mathbf{2 4} & \mathbf{2 1} & \mathbf{4} & \mathbf{5} & \mathbf{5 5} & \mathbf{0} .84 \\ - & 2 & - & - & - & - & - & - & 2 & 0.03 \\ - & 11 & - & - & - & - & - & - & 11 & 0.17 \\ - & - & 1 & - & - & - & - & 3 & 4 & 0.06 \\ 1 & 5 & 2 & 3 & - & - & - & - & 11 & 0.17\end{array}$


Table 1, continued

M. hilaris Schmitz, 1927

M. hirticrus (Schmitz, 1918)

M. hyalipennis (Wood, 1912)

M. ignobilis (Schmitz, 1919)

M. insons (Lundbeck, 1920)

M. jameslamonti (Disney, 1995)

M. latifrons (Wood, 1910)

M. latior Schmitz, 1936

M. limburgensis (Schmitz, 1918)

M. longipalpis (Wood, 1910)

M. lucifrons (Schmitz, 1918)

M. Iutea (Meigen, 1830)

M. luteipes (Schmitz, 1918)

M. major (Wood, 1912)

M. maura (Wood, 1910)

M. meconicera (Speiser, 1925)

M. minor (Zetterstedt, 1848)

M. nasoni (Malloch, 1914)

M. nigra (Meigen, 1830)

M. nigriceps (Loew, 1866)

M. obscuripennis (Wood, 1909)

M. oweni Disney, 1988

M. pectoralis (Wood, 1910)

M. pectorella Schmitz, 1929

M. pleuralis (Wood, 1909)

M. plurispinulosa (Zetterstedt, 1860)

M. propinqua (Wood, 1909)

M. protarsalis Schmitz, 1927

M. pulicaria -complex

M. pumila (Meigen, 1830)

M. pusilla (Meigen, 1830)

M. quadriseta (Schmitz, 1918)

M. rubella (Schmitz, 1920)

M. rubescens (Wood, 1912)

$M$. rufipes (Meigen, 1804)

M. setulipalpis Schmitz, 1938

M. simulans (Wood, 1912)

M. sordida (Zetterstedt, 1838)

M. speiseri Schmitz, 1929

M. spinata (Wood, 1910)

M. spinicincta (Wood, 1910)

M. spinigera (Wood, 1908)

M. stichata (Lundbeck, 1920)

M. striolata Schmitz, 1940

M. subcarpalis (Lundbeck, 1920)

M. subpalpalis (Lundbeck, 1920)

M. subpleuralis (Wood, 1909)

M. sulphuripes (Wood, 1910)

M. sylvatica (Wood, 1910)

M. tama (Schmitz, 1926)

M. tarsalis (Wood, 1910)

M. unicolor (Schmitz, 1919)

M. variana Schmitz, 1926

M. woodi (Lundbeck, 1922)

M. xanthozona (Strobl, 1892)

Megaselia sp. males

*Menozziola obscuripes (Schmitz, 1927) -

\begin{tabular}{|c|c|c|c|c|c|c|c|c|}
\hline 3 & - & - & - & - & - & - & 3 & 0.05 \\
\hline 39 & - & - & - & - & - & 1 & 43 & 0.66 \\
\hline 1 & - & 11 & 18 & 34 & 3 & - & 67 & 1.02 \\
\hline 12 & 4 & 2 & 1 & - & - & - & 19 & 0.29 \\
\hline 5 & 11 & 8 & 21 & 72 & - & 2 & 119 & 1.81 \\
\hline 1 & - & - & - & - & - & - & 1 & 0.02 \\
\hline- & 2 & - & - & 2 & - & - & 4 & 0.06 \\
\hline 4 & - & - & - & - & - & - & 4 & 0.06 \\
\hline 46 & - & - & - & - & - & 1 & 47 & 0.72 \\
\hline 2 & - & - & - & - & - & - & 2 & 0.03 \\
\hline 17 & 4 & - & 1 & 1 & - & - & 27 & 0.41 \\
\hline 6 & - & - & 3 & 11 & - & - & 20 & 0.30 \\
\hline 4 & - & - & - & - & - & - & 6 & 0.09 \\
\hline 1 & - & - & - & - & - & 4 & 5 & 0.08 \\
\hline 6 & - & - & - & - & - & - & 6 & 0.09 \\
\hline 3 & 5 & - & - & - & 3 & 8 & 19 & 0.29 \\
\hline- & 1 & - & - & - & - & - & 1 & 0.02 \\
\hline 3 & - & - & - & - & - & - & 3 & 0.05 \\
\hline 1 & 1 & - & - & - & - & - & 2 & 0.03 \\
\hline 380 & 172 & 50 & 57 & 88 & 49 & 72 & 868 & 13.22 \\
\hline- & - & - & - & 3 & - & - & 3 & 0.05 \\
\hline- & - & - & 6 & 1 & 6 & 2 & 15 & 0.23 \\
\hline 86 & 2 & 2 & 9 & 1 & 3 & 2 & 105 & 1.60 \\
\hline 285 & 51 & - & - & - & - & - & 336 & 5.12 \\
\hline 156 & 941 & 17 & 393 & 301 & 66 & 58 & 1932 & 29.43 \\
\hline 26 & - & 2 & 3 & 11 & - & 2 & 44 & 0.67 \\
\hline 1 & - & - & - & - & - & - & 1 & 0.02 \\
\hline- & - & - & - & 1 & - & - & 1 & 0.02 \\
\hline 40 & 12 & 5 & 141 & 162 & 79 & 62 & 508 & 7.74 \\
\hline 116 & 64 & 28 & 1 & 3 & 2 & 1 & 217 & 3.31 \\
\hline 110 & 50 & 6 & 1 & - & 6 & 4 & 177 & 2.70 \\
\hline 16 & 2 & - & - & 2 & 2 & - & 23 & 0.35 \\
\hline 5 & 1 & - & 1 & 4 & - & - & 11 & 0.17 \\
\hline 2 & - & - & - & - & - & - & 2 & 0.03 \\
\hline- & - & - & 2 & 1 & - & - & 3 & 0.05 \\
\hline 34 & 7 & 5 & 1 & 6 & - & 4 & 57 & 0.87 \\
\hline 1 & - & - & - & 1 & - & - & 2 & 0.03 \\
\hline 4 & - & - & - & - & - & - & 4 & 0.06 \\
\hline- & 1 & - & - & - & - & - & 1 & 0.02 \\
\hline 6 & - & - & - & - & - & - & 6 & 0.09 \\
\hline 2 & 1 & 1 & - & - & - & - & 9 & 0.14 \\
\hline 1 & - & - & - & - & - & - & 2 & 0.03 \\
\hline- & - & - & 1 & - & - & - & 1 & 0.02 \\
\hline 8 & 4 & 2 & 2 & 7 & 2 & - & 26 & 0.40 \\
\hline- & - & 2 & - & 1 & - & - & 3 & 0.05 \\
\hline- & - & 1 & 2 & - & 5 & 2 & 10 & 0.15 \\
\hline 14 & - & 3 & 3 & 16 & 7 & 12 & 55 & 0.84 \\
\hline 2 & 1 & 3 & - & - & - & - & 6 & 0.09 \\
\hline 3 & - & 2 & 4 & 2 & 1 & 6 & 18 & 0.27 \\
\hline- & 1 & - & 1 & - & - & - & 2 & 0.03 \\
\hline 3 & - & - & - & - & - & - & 3 & 0.05 \\
\hline- & - & - & 80 & 167 & 17 & 70 & 334 & 5.09 \\
\hline 10 & 4 & 4 & - & - & - & - & 19 & 0.29 \\
\hline- & - & - & - & 2 & 5 & 5 & 12 & 0.18 \\
\hline 1 & 6 & 2 & - & - & - & - & 9 & 0.14 \\
\hline 76 & 21 & 7 & 30 & 33 & 4 & 7 & 185 & 2.82 \\
\hline 83 & 70 & - & - & - & - & - & 153 & \\
\hline
\end{tabular}


Table 1, continued

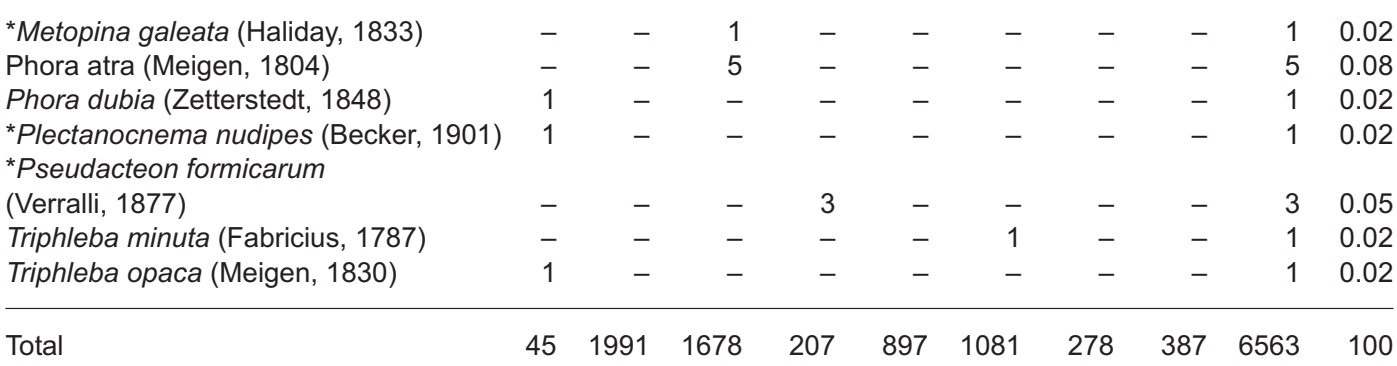

selia spp., and were not included in the analysis. All material from this study is deposited at the Swedish Museum of Natural History, Stockholm.

\subsection{Study sites}

The most common type of habitat before the large wildfire was mature pine forest on bedrock, constituting more than $50 \%$ of the total area. Then follows spruce-dominated forest on the slopes and bottoms of the fissure-valleys (30\%), wetland $(11 \%)$, mixed forest $(6 \%)$, and deciduous forest (2\%) (Petterson 2006).

All traps considered in this study were situated on burnt mature pine forests on bedrock, and the intensity of the fires ranged from weak to medium, with the associated destruction of the humus layer of the soil ranging from moderate to deep. The intensity of fire is strong when the crowns of the trees are dead and weak when these are not affected by the fire and therefore still green (i.e. flame height ranging from weak, moderate, intermediate to strong). Within the major wildfire area, the smoldering fires in the humus destroyed most of the organic soil in some parts (i.e., effect of the soil ranging from weak, moderate, intermediate to strong). Four traps were deployed on three wildfire sites A, B and C (Fig. 1) and were emptied at intervals of 24 to 49 days (for further details see Table 1). In the following, the sites are described in more details.

- Site A: medium-sized (20 ha) and dominated by Pinus-Betula tree species. The fire occurred on the $6^{\text {th }}$ of June 1997. One trap (nr 1: samples 27 and 29) was deployed two years after the fire on a spot that had been exposed to intermediate fire intensity and soil destruction.

- Site B: small-sized (2-3 ha) and dominated by Pinus-Picea tree species. The fire occurred on the $1^{\text {st }}$ of June 1999. One trap (nr 2: samples 9 and 10) was deployed 10 days after the fire on a spot that had been exposed to intermediate fire intensity and moderate soil destruction.

- Site C: large-sized (450 ha) and dominated by Pinus-Betula tree species. The fire occurred between 1-12 August 1999. Two traps (nr 3 and 4: samples 17-20) were deployed close to each other directly after the fire on spots that had been exposed to intermediate fire intensity and strong soil destruction.

\subsection{Data analysis}

Analyses were based solely on male individuals, as most females of Megaselia spp. and Phora spp. are not identifiable to species level, consequently, these have only been identified to genus level. We described the scuttle fly assemblages by dominance structures and the relative abundances of species for each site. We classed species as "dominant" if its relative abundance was $\geq 1 \%$.

We compared seasonal changes (phenology) in abundance ( $n$-number of specimens) and species richness ( $s-$ number of species). Since the sampling effort varied between periods, we standardized the number of active sampling days to 20 [(actual number of individuals collected/number of days of active sampling) $\times 20$, calculated in Microsoft Excel 2003] to make the number of individuals comparable. To correct species richness for the influence of different sampling efforts between the samples, we used a non-para- 
Table 2. List of species (males) with the number of individuals standardized to 20 trap days (based on Table 1) [(number of individuals collected/number of trap days) $\times 20$ days]. Relative abundance $(\%)$ is given for the total sample. Dominant species for the equal sampling effort with dominance $\geq 1 \%$ are shown in bold type. T.S $=$ total sample. Species with * are new to Sweden.

\begin{tabular}{|c|c|c|c|c|c|c|c|c|c|c|}
\hline Sample & 29 & 27 & 9 & 10 & 17 & 19 & 18 & 20 & T.S & $S(\%)$ \\
\hline Anevrina unispinosa & - & 0.82 & - & - & - & - & - & - & 0.82 & 0.02 \\
\hline${ }^{*}$ Beckerina umbrimargo & _- & 0.41 & - & _- & - & - & - & - & 0.41 & 0.01 \\
\hline Conicera floricola & - & - & 1.08 & - & - & - & - & - & 1.08 & 0.03 \\
\hline Conicera similis & - & - & 1.62 & - & - & - & - & - & 1.62 & 0.05 \\
\hline Conicera tibialis & - & - & 1.08 & - & - & - & - & - & 1.08 & 0.03 \\
\hline Diplonevra nitidula & - & - & 0.54 & - & 12.86 & - & - & 0.95 & 14.35 & 0.40 \\
\hline Hypocerides nearcticus & - & - & - & - & 2.14 & - & - & - & 2.14 & 0.06 \\
\hline Megaselia abdita & - & - & 0.54 & - & - & - & - & - & 0.54 & 0.02 \\
\hline M. aculeata & - & - & - & - & - & - & 0.48 & - & 0.48 & 0.01 \\
\hline M. aequalis & - & 0.41 & - & - & - & - & - & - & 0.41 & 0.01 \\
\hline M. affinis & - & 0.41 & 2.70 & 2.27 & - & - & - & - & 5.38 & 0.15 \\
\hline M. albicaudata & - & 2.04 & - & - & - & - & - & - & 2.04 & 0.06 \\
\hline M. albiclava & - & 1.22 & - & - & - & - & - & - & 1.22 & 0.03 \\
\hline M. albocingula & - & 4.08 & 2.70 & 1.36 & 2.86 & 14.29 & 0.48 & 0.48 & 26.24 & 0.73 \\
\hline M. annulipes & - & 0.41 & - & - & - & - & - & - & 0.41 & 0.01 \\
\hline M. barbulata & - & - & - & - & - & 0.71 & - & - & 0.71 & 0.02 \\
\hline M. basispinata & - & 5.71 & 10.27 & 0.91 & 2.14 & 6.43 & - & - & 25.47 & 0.71 \\
\hline M. berndseni & - & 0.82 & 2.16 & - & 0.71 & - & - & - & 3.69 & 0.10 \\
\hline M. bifida & - & 0.41 & - & - & 0.71 & 0.71 & - & - & 1.84 & 0.05 \\
\hline M. brevicostalis & - & 51.02 & 100.00 & 7.73 & 34.29 & 46.43 & - & 3.81 & 243.27 & 6.79 \\
\hline M. campestris & - & - & - & 0.45 & - & - & - & - & 0.45 & 0.01 \\
\hline M. ciliata & - & 1.22 & - & - & - & - & - & - & 1.22 & 0.03 \\
\hline M. communiformis & - & 0.82 & - & - & - & - & - & - & 0.82 & 0.02 \\
\hline M. conformis & 1.67 & 0.41 & - & - & - & - & - & - & 2.07 & 0.06 \\
\hline M. consetigera & - & 5.31 & - & - & - & - & - & - & 5.31 & 0.15 \\
\hline M. crassipes & - & 0.41 & - & - & - & - & - & - & 0.41 & 0.01 \\
\hline M. curvicapilla & 2.50 & 11.02 & 0.54 & - & - & - & - & - & 14.06 & 0.39 \\
\hline M. differens & - & 2.45 & - & - & - & - & - & - & 2.45 & 0.07 \\
\hline M. discreta & - & 2.04 & - & 0.45 & 2.86 & 0.71 & - & - & 6.07 & 0.17 \\
\hline M. diversa & - & 7.35 & - & - & 6.43 & 13.57 & 3.33 & 8.57 & 39.25 & 1.10 \\
\hline M. drakei & - & 2.45 & - & 0.91 & - & - & - & - & 3.36 & 0.09 \\
\hline M. eccoptomera & - & 0.82 & - & - & - & - & - & - & 0.82 & 0.02 \\
\hline M. elongata & - & - & 0.54 & - & - & - & - & - & 0.54 & 0.02 \\
\hline M. emarginata & - & 8.16 & - & 0.45 & - & - & - & - & 8.62 & 0.24 \\
\hline M. excavata & - & - & - & 2.27 & - & 5.00 & 2.38 & 9.05 & 18.70 & 0.52 \\
\hline M. flavescens & - & 0.41 & - & - & - & - & - & - & 0.41 & 0.01 \\
\hline M. flavicans & 1.67 & 0.82 & - & - & - & - & - & - & 2.48 & 0.07 \\
\hline M. frameata & - & 3.27 & 0.54 & - & - & 0.71 & - & 0.48 & 5.00 & 0.14 \\
\hline M. fungivora & - & 23.67 & - & - & - & - & - & 0.48 & 24.15 & 0.67 \\
\hline M. furva & - & 0.41 & - & 0.45 & - & 1.43 & - & 1.90 & 4.20 & 0.12 \\
\hline M. fuscipe & - & 1.22 & - & - & - & - & - & - & 1.22 & 0.03 \\
\hline M. fuscoides & - & 0.41 & - & - & - & - & - & - & 0.41 & 0.01 \\
\hline M. fuscovarian & - & 0.41 & - & - & - & - & - & - & 0.41 & 0.01 \\
\hline M. gartensis & - & 0.41 & - & - & - & - & - & - & 0.41 & 0.01 \\
\hline M. giraudii -compl. & 0.41 & - & - & 17.14 & 15.00 & 1.90 & 2.38 & 36.84 & 1.03 & \\
\hline M. glabrifrons & - & 0.82 & - & - & - & - & - & - & 0.82 & 0.02 \\
\hline M. gregaria & - & 4.49 & - & - & - & - & - & - & 4.49 & 0.13 \\
\hline${ }^{*} M$. henrydisneyi & - & - & 0.54 & - & - & - & - & 1.43 & 1.97 & 0.05 \\
\hline M. hibernans & 0.83 & 2.04 & 1.08 & 1.36 & - & - & - & - & 5.32 & 0.15 \\
\hline M. hilaris & - & 1.22 & - & - & - & - & - & - & 1.22 & 0.03 \\
\hline M. hirticrus & 2.50 & 15.92 & - & - & - & - & - & 0.48 & 18.89 & 0.53 \\
\hline M. hyalipennis & - & 0.41 & - & 5.00 & 12.86 & 24.29 & 1.43 & - & 43.98 & 1.23 \\
\hline M. ignobilis & - & 4.90 & 2.16 & 0.91 & 0.71 & - & - & - & 8.68 & 0.24 \\
\hline M. insons & - & 2.04 & 5.95 & 3.64 & 15.00 & 51.43 & - & 0.95 & 79.00 & 2.21 \\
\hline M. jameslamonti & - & 0.41 & - & - & - & - & - & - & 0.41 & 0.01 \\
\hline
\end{tabular}


Table 2, continued

\begin{tabular}{|c|c|c|c|c|c|c|c|c|c|c|}
\hline M. latifrons & - & - & 1.08 & - & - & 1.43 & - & - & 2.51 & 0.07 \\
\hline M. latior & - & 1.63 & - & - & - & - & - & - & 1.63 & 0.05 \\
\hline M. limburgensis & - & 18.78 & - & - & - & - & - & 0.48 & 19.25 & 0.54 \\
\hline M. longipalpis & - & 0.82 & - & - & - & - & - & - & 0.82 & 0.02 \\
\hline M. lucifrons & 3.33 & 6.94 & 2.16 & - & 0.71 & 0.71 & - & - & 13.86 & 0.39 \\
\hline M. lutea & - & 2.45 & - & - & 2.14 & 7.86 & - & - & 12.45 & 0.35 \\
\hline M. luteipes & 1.67 & 1.63 & - & - & - & - & - & - & 3.30 & 0.09 \\
\hline M. major & - & 0.41 & - & - & - & - & - & 1.90 & 2.31 & 0.06 \\
\hline M. maura & - & 2.45 & - & - & - & - & - & - & 2.45 & 0.07 \\
\hline M. meconicera & - & 1.22 & 2.70 & - & - & - & 1.43 & 3.81 & 9.17 & 0.26 \\
\hline M. minor & - & - & 0.54 & - & - & - & - & - & 0.54 & 0.02 \\
\hline M. nasoni & - & 1.22 & - & - & - & - & - & - & 1.22 & 0.03 \\
\hline M. nigra & - & 0.41 & 0.54 & - & - & - & - & - & 0.95 & 0.03 \\
\hline M. nigriceps & - & 155.10 & 92.97 & 22.73 & 40.71 & 62.86 & 23.33 & 34.29 & 431.99 & 12.07 \\
\hline M. obscuripennis & - & - & - & - & - & 2.14 & - & - & 2.14 & 0.06 \\
\hline M. oweni & - & - & - & - & 4.29 & 0.71 & 2.86 & 0.95 & 8.81 & 0.25 \\
\hline M. pectoralis & - & 35.10 & 1.08 & 0.91 & 6.43 & 0.71 & 1.43 & 0.95 & 46.62 & 1.30 \\
\hline M. pectorella & - & 116.33 & 27.57 & - & - & - & - & - & 143.89 & 4.02 \\
\hline M. pleuralis & - & 63.67 & 508.65 & 7.73 & 280.71 & 215.00 & 31.43 & 27.62 & 1134.81 & 31.70 \\
\hline M. plurispinulosa & - & 10.61 & - & 0.91 & 2.14 & 7.86 & - & 0.95 & 22.47 & 0.63 \\
\hline M. propinqua & - & 0.41 & - & - & - & - & - & - & 0.41 & 0.01 \\
\hline M. protarsalis & - & - & - & - & - & 0.71 & - & - & 0.71 & 0.02 \\
\hline M. pulicaria-comp. & 5.83 & 16.33 & 6.49 & 2.27 & 100.71 & 115.71 & 37.62 & 29.52 & 314.49 & 8.78 \\
\hline M. pumila & 1.67 & 47.35 & 34.59 & 12.73 & 0.71 & 2.14 & 0.95 & 0.48 & 100.62 & 2.81 \\
\hline M. pusilla & - & 44.90 & 27.03 & 2.73 & 0.71 & - & 2.86 & 1.90 & 80.13 & 2.24 \\
\hline M. quadriseta & 0.83 & 6.53 & 1.08 & - & - & 1.43 & 0.95 & - & 10.83 & 0.30 \\
\hline M. rubella & - & 2.04 & 0.54 & - & 0.71 & 2.86 & - & - & 6.15 & 0.17 \\
\hline M. rubescens & - & 0.82 & - & - & - & - & - & - & 0.82 & 0.02 \\
\hline M. rufipes & - & - & - & - & 1.43 & 0.71 & - & - & 2.14 & 0.06 \\
\hline M. setulipalpis & - & 13.88 & 3.78 & 2.27 & 0.71 & 4.29 & - & 1.90 & 26.84 & 0.75 \\
\hline M. simulans & - & 0.41 & - & - & - & 0.71 & - & - & 1.12 & 0.03 \\
\hline M. sordida & - & 1.63 & - & - & - & - & - & - & 1.63 & 0.05 \\
\hline M. speiseri & - & - & 0.54 & - & - & - & - & - & 0.54 & 0.02 \\
\hline M. spinata & - & 2.45 & - & - & - & - & - & - & 2.45 & 0.07 \\
\hline M. spinicincta & 4.17 & 0.82 & 0.54 & 0.45 & - & - & - & - & 5.98 & 0.17 \\
\hline M. spinigera & 0.83 & 0.41 & - & - & - & - & - & - & 1.24 & 0.03 \\
\hline M. stichata & - & - & - & - & 0.71 & - & - & - & 0.71 & 0.02 \\
\hline M. striolata & 0.83 & 3.27 & 2.16 & 0.91 & 1.43 & 5.00 & 0.95 & - & 14.55 & 0.41 \\
\hline M. subcarpalis & - & - & - & 0.91 & - & 0.71 & - & - & 1.62 & 0.05 \\
\hline M. subpalpalis & - & - & - & 0.45 & 1.43 & - & 2.38 & 0.95 & 5.22 & 0.15 \\
\hline M. subpleuralis & - & 5.71 & - & 1.36 & 2.14 & 11.43 & 3.33 & 5.71 & 29.70 & 0.83 \\
\hline M. sulphuripes & - & 0.82 & 0.54 & 1.36 & - & - & - & - & 2.72 & 0.08 \\
\hline M. sylvatica & - & 1.22 & - & 0.91 & 2.86 & 1.43 & 0.48 & 2.86 & 9.75 & 0.27 \\
\hline M. tama & - & - & 0.54 & - & 0.71 & - & - & - & 1.25 & 0.04 \\
\hline M. tarsalis & - & 1.22 & - & - & - & - & - & - & 1.22 & 0.03 \\
\hline M. unicolor & - & - & - & - & 57.14 & 119.29 & 8.10 & 33.33 & 217.86 & 6.08 \\
\hline M. variana & 0.83 & 4.08 & 2.16 & 1.82 & - & - & - & - & 8.90 & 0.25 \\
\hline M. woodi & - & - & - & - & - & 1.43 & 2.38 & 2.38 & 6.19 & 0.17 \\
\hline M. xanthozona & - & 0.41 & 3.24 & 0.91 & - & - & - & - & 4.56 & 0.13 \\
\hline Megaselia sp. males & 5.83 & 31.02 & 11.35 & 3.18 & 21.43 & 23.57 & 1.90 & 3.33 & 101.63 & 2.84 \\
\hline *Menozziola obscuripes & - & 33.88 & 37.84 & - & - & - & - & - & 71.72 & 2.00 \\
\hline *Metopina galeata & - & - & 0.54 & - & - & - & - & - & 0.54 & 0.02 \\
\hline Phora atra & - & - & 2.70 & - & - & - & - & - & 2.70 & 0.08 \\
\hline Phora dubia & 0.83 & - & - & - & - & - & - & - & 0.83 & 0.02 \\
\hline${ }^{*}$ Plectanocnema nudipes & 0.83 & - & - & - & - & - & - & - & 0.83 & 0.02 \\
\hline${ }^{*}$ Pseudacteon formicarum & - & - & - & 1.36 & - & - & - & - & 1.36 & 0.04 \\
\hline Triphleba minuta & - & - & - & - & - & 0.71 & - & - & 0.71 & 0.02 \\
\hline Triphleba opaca & 0.83 & - & - & - & - & - & - & - & 0.83 & 0.02 \\
\hline ota & 37.5 & 812.2 & 907.0 & 94.1 & 640.7 & 772.1 & 132.4 & 184.3 & 3580.4 & 10 \\
\hline
\end{tabular}


Table 3. Sampling periods and summary values of number of species of the scuttle fly assemblages of the Tyresta Forest after wildfires. Sobs = species observed, Chao1 = Chao1 estimate, S.D. = standard deviation. Letters in parenthesis refer to settings in Fig. 1.

\begin{tabular}{|c|c|c|c|c|c|c|c|c|c|}
\hline Sample & $29(\mathrm{~A})$ & $27(\mathrm{~A})$ & $9(\mathrm{~B})$ & $10(\mathrm{~B})$ & $17(\mathrm{C})$ & $19(\mathrm{C})$ & $18(\mathrm{C})$ & $20(C)$ & Total \\
\hline Period & $\begin{array}{c}05 . \mathrm{V}- \\
28 . \mathrm{V}\end{array}$ & $\begin{array}{l}\text { 28.V- } \\
\text { 15.VII }\end{array}$ & $\begin{array}{l}\text { 11.VI- } \\
\text { 17.VII }\end{array}$ & $\begin{array}{l}\text { 17.VII- } \\
29 . \text { VIII }\end{array}$ & $\begin{array}{l}\text { 13.VIII- } \\
9 . \mathrm{IX}\end{array}$ & $\begin{array}{l}\text { 13.VIII- } \\
9 . \mathrm{IX}\end{array}$ & $\begin{array}{l}9.1 X- \\
20 . X\end{array}$ & $\begin{array}{l}9.1 X- \\
20 . X\end{array}$ & \\
\hline Sobs & 17 & 80 & 42 & 32 & 33 & 37 & 21 & 29 & 112 \\
\hline Singletons & 8 & 21 & 14 & 6 & 10 & 12 & 3 & 6 & 24 \\
\hline Doubletons & 4 & 10 & 6 & 9 & 3 & 5 & 3 & 6 & 12 \\
\hline Chao1 & 25 & 102 & 58 & 34 & 50 & 51 & 23 & 32 & 136 \\
\hline S.D. & 7.5 & 12.8 & 11.7 & 2.3 & 14.8 & 11.2 & 2.3 & 3.2 & 12.9 \\
\hline
\end{tabular}

Table 4. Summary values of observed species richness, species richness estimate and trap days from different localities. Sobs = species observed, Chao1 = Chao 1 estimate, S.D. $=$ standard deviation.

\begin{tabular}{lrrrc}
\hline & Sobs & Chao1 & S.D. & Trap days \\
\hline Tyresta Forest & 112 & 136 & 12.96 & 294 \\
Ticino Forest & 63 & 77 & 8.43 & $161\left(483^{\mathrm{a}}\right)$ \\
Białowieża Primeval Forest & 75 & 95 & 11.83 & 358 \\
Tuchola Forest & 77 & 106 & 15.81 & 358 \\
Biała Forest & 52 & 63 & 7.67 & 358 \\
Pisz Forest & 72 & 84 & 8.81 & 118 \\
\hline
\end{tabular}

a) 483 trap days including all three traps on each site (table 2 in Prescher et al. 2002)

Table 5. Percentage of dominants $(n \geq 1 \%)$ on each site after wildfires in 1997 and 1999. All sampling was made in 1999. Letters in parenthesis indicate settings in Fig. 1.

\begin{tabular}{|c|c|c|c|c|c|c|c|c|}
\hline \multirow[t]{2}{*}{ Species } & \multicolumn{2}{|l|}{1997} & \multicolumn{6}{|l|}{1999} \\
\hline & $\begin{array}{c}29(\mathrm{~A}) \\
1999\end{array}$ & $\begin{array}{c}27(\mathrm{~A}) \\
1999\end{array}$ & $\begin{array}{l}9(\mathrm{~B}) \\
1999\end{array}$ & $\begin{array}{c}10(B) \\
1999\end{array}$ & $\begin{array}{c}17(\mathrm{C}) \\
1999\end{array}$ & $\begin{array}{c}19(\mathrm{C}) \\
1999\end{array}$ & $\begin{array}{c}18(\mathrm{C}) \\
1999\end{array}$ & $\begin{array}{c}20(C) \\
1999\end{array}$ \\
\hline Megaselia pleuralis & - & 7.84 & 56.08 & 8.21 & 43.81 & 27.84 & 23.74 & 14.99 \\
\hline Megaselia nigriceps & - & 19.09 & 10.25 & 24.15 & 6.35 & 8.14 & 17.63 & 18.60 \\
\hline Megaselia pulicaria-compl. & 15.56 & 2.01 & 0.72 & 2.42 & 15.72 & 14.99 & 28.42 & 16.02 \\
\hline Megaselia brevicostalis & - & 6.28 & 11.03 & 8.21 & 5.35 & 6.01 & - & 2.07 \\
\hline Megaselia unicolor & - & - & - & - & 8.92 & 15.45 & 6.12 & 18.09 \\
\hline Megaselia pectorella & - & 14.31 & 3.04 & - & - & - & - & - \\
\hline Megaselia pumila & 4.44 & 5.83 & 3.81 & 13.53 & 0.11 & 0.28 & 0.72 & 0.26 \\
\hline Megaselia pusilla & - & 5.52 & 2.98 & 2.90 & 0.11 & - & 2.16 & 1.03 \\
\hline Megaselia insons & - & 0.25 & 0.66 & 3.86 & 2.34 & 6.66 & - & 0.52 \\
\hline Menozziola obscuripes & - & 4.17 & 4.17 & - & - & - & - & - \\
\hline Megaselia pectoralis & - & 4.32 & 0.12 & 0.97 & 1.00 & 0.09 & 1.08 & 0.52 \\
\hline Megaselia hyalipennis & - & 0.05 & - & 5.31 & 2.01 & 3.15 & 1.08 & - \\
\hline Megaselia diversa & - & 0.90 & - & - & 1.00 & 1.76 & 2.52 & 4.65 \\
\hline Megaselia giraudii-compl. & - & 0.05 & - & - & 2.68 & 1.94 & 1.44 & 1.29 \\
\hline Total & 20.00 & 71.57 & 92.86 & 69.56 & 86.72 & 84.37 & 83.47 & 76.75 \\
\hline
\end{tabular}




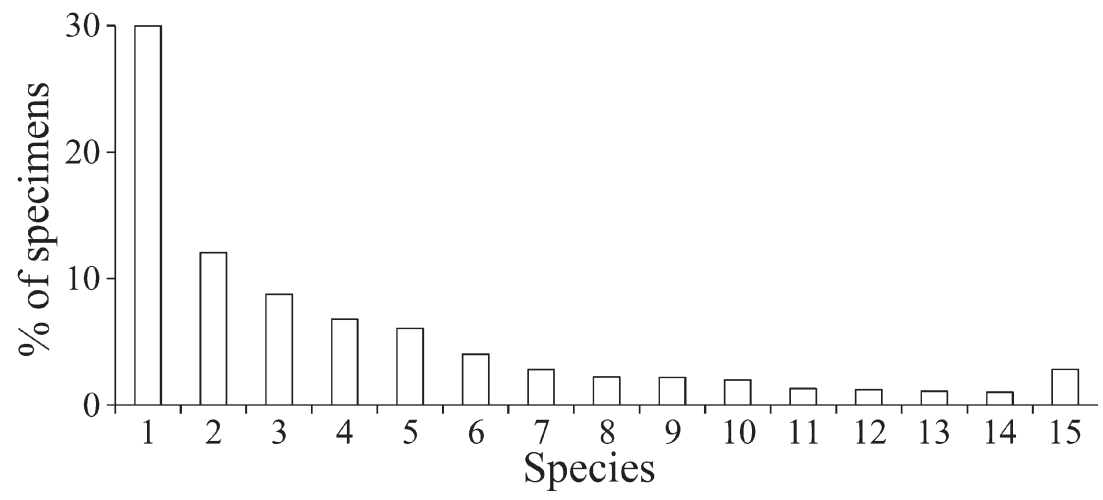

Fig. 2. Percentage of species representing $\geq 1 \%$ of scuttle flies collected in 1999 , all samples, standardized to a sampling effort of 20 days. Numbers on the $x$-axis refer to the names of dominant species: 1 - Megaselia pleuralis, 2 - Megaselia nigriceps, 3 Megaselia pulicaria-complex, 4 - Megaselia brevicostalis, 5 - Megaselia unicolor, 6 Megaselia pectorella, 7 - Megaselia pumila, 8 - Megaselia pusilla, 9 - Megaselia insons, 10 - Menozziola obscuripes, 11 - Megaselia pectoralis, 12 - Megaselia hyalipennis, 13 - Megaselia diversa, 14 - M. giraudii-complex, 15 - Megaselia sp. males.

metric abundance-based species richness estimator, Chaol (Chao 1984, 1987, Chao \& Shen 2003). Species richness estimations and statistics were made in SPADE (Chao \& Shen 2003).

\section{Results}

We identified 12 genera and 112 species from a total of 8,051 individuals (i.e., including both males and females of scuttle flies). Six species were new to Sweden (Tables $1 \& 2$ ).

\subsection{Assemblage structure}

Individuals of species in the genus Megaselia (i.e. 7,816 specimens) constituted from $93 \%$ to $100 \%$ of the scuttle fly assemblage associated with each site. We restricted our analyses to males identified to species level (i.e. 6,563 specimens), which constituted over $80 \%$ of all individuals collected (Table 1). The total species richness (identified number of species) of the scuttle fly assemblage of Tyresta in 1999 after the wildfires was rather high $(s=112$, Chao $1=136$, S.D. $=12.9$, trap days $=294)$ (Tables $3 \& 4)$.

The numbers of species amongst samples varied from $s=17$ (sample 29) to $s=80$ (sample 27), whereas species richness estimates for the same samples, varied from $s=25$ to $s=102$ (Table 3). Fourteen of the 112 species collected at Tyresta were considered to be dominant (sum of abundances of all samples $\geq 1 \%$ ): Megaselia pleuralis, $M$. nigriceps, $M$. pulicaria-complex, M. brevicostalis, M. pectorella, M. unicolor, M. pumila, M. pusilla, Menozziola obscuripes, Megaselia insons, $M$. pectoralis, $M$. hyalinipennis, $M$. diversa and $M$. giraudii-complex (Table 2; Fig. 2). Dominant species per sample and per site are presented in Table 5.

\subsection{Phenology}

We observed two peaks of abundance: in the spring/summer (sample 27) (May to June), and in summer/autumn (sample 19) (August to September - the smaller one) (Fig. 3). Chao 1 estimate slightly altered the number of species compared with observed (lower) number of species (Fig. 3).

The most abundant species of the dominant group (i.e. Megaselia pleuralis, $M$. nigriceps, $M$. pulicaria-complex and $M$. brevicostalis) are multivoltine, displaying spring and late summer/autumn activity. The majority of the dominant scuttle fly species in Tyresta with known diet preferences are saprophagous species (Table 6). 


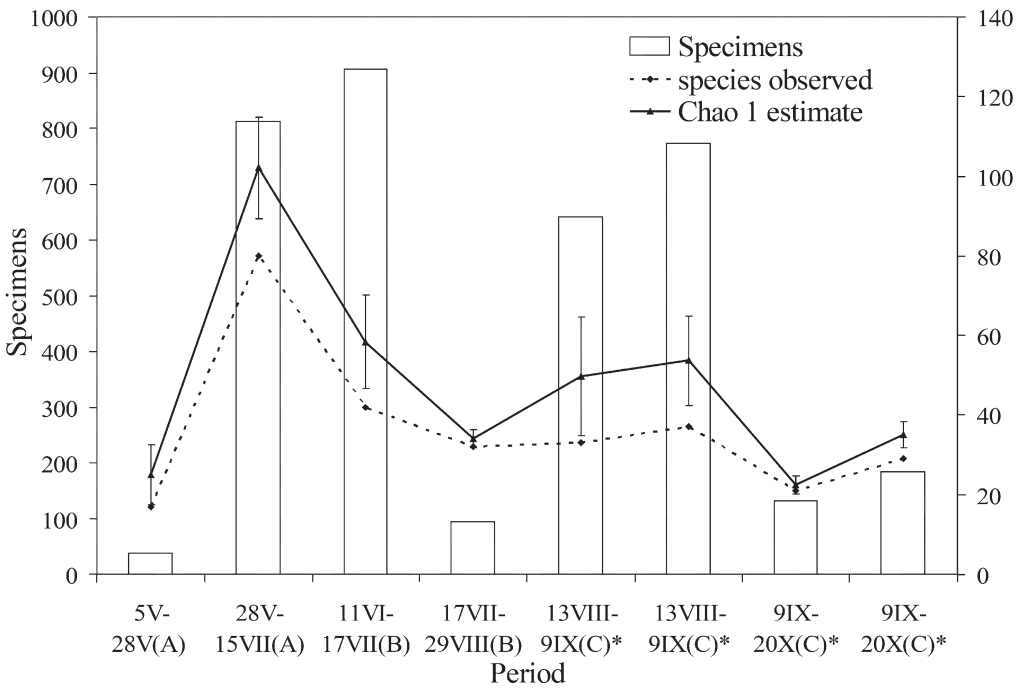

Fig. 3. Seasonal changes in abundance and species richness (number of species based on observed and estimated numbers Chao1) of adult scuttle flies caught in Malaise traps in 1999. Error bars signify standard deviation. Letters in parenthesis refer to sites in Figure 1. *: same time periods due to use of two traps on the same location (double traps).
Of the species listed in Tables $1 \& 214.0 \%$ are known to be saprophages, $16.7 \%$ fungivores, $7 \%$ parasitoids or specialized predators and $62.3 \%$ of unknown larval habits.

\section{Discussion}

Our study reconfirms the results of previous work showing that Megaselia species make a major contribution to the assemblages of Phoridae, especially after disturbance or stress (Disney 2004,
Durska 1996, 2001, 2006, 2009). The same result was obtained by Prescher et al. (2002) using emergence traps located in the chestnut belt following a wildfire in the Swiss Alps where Megaselia accounted for over $90 \%$ of all Phoridae sampled.

One major, surprising difference was the absence of Megaselia verralli in our Tyresta samples. This species of unknown biology was a eudominant in the Ticino Forest and also a eudominant and a characteristic species of the pine plantations in the Polish Lowland: Biało-

Table 6. Trophic-group and male activity of the dominant species ( $n \geq 1$, equal sampling effort) of scuttle flies in the Tyresta Forest after wildfires with percent number of collected specimens. ? = unknown (or probable) diet of larvae.

\begin{tabular}{lrll}
\hline $\begin{array}{l}\text { Species } \\
\text { activity }\end{array}$ & $\%$ & Trophic groups & Time peaks of male \\
\hline Megaselia nigriceps & 12.07 & Saprophagous & \\
Megaselia pulicaria-complex & 8.78 & Polysaprophagous & V-VII, VIII-IX \\
Megaselia brevicostalis & 6.79 & Polysaprophagous & V-VII, VIII-IX \\
Megaselia unicolor & 6.08 & Saprophagous & VIII-IX \\
Megaselia pectorella & 4.02 & Saprophagous & V-VII \\
Megaselia pumila & 3.31 & $?$ & V-VII \\
Megaselia pusilla & 2.81 & $?$ & V-VII \\
Megaselia insons & 2.21 & $?$ & VIII-IX \\
Menozziola obscuripes & 2.00 & Parasitoids on ants & V-VII \\
Megaselia pectoralis & 1.30 & Saprophagous & V-VII \\
Megaselia hyalipennis & 1.23 & Fungiphagous & VIII-IX \\
Megaselia diversa & 1.10 & Saprophagous (?) & V-VIII III-IX \\
Megaselia giraudii-complex & 1.03 & Polysaprophagous & V-VII, VIII-IX \\
\end{tabular}


wieża Forest, Tuchola Forest and Biała Forest. In the Ticino Forest, Phoridae were collected using emergence traps, but by yellow pan traps in pine plantations of the Polish Lowland (Durska 2001, 2006, 2009, Prescher et al. 2002). M. albocingulata, which belongs to the $M$. verralli group, was caught at all three wildfire localities in Tyresta Forest and is a dominant species in samples 10 and 19 (Table 5). This species (M. albocingulata) has not been found in the Ticino Forest but is known from wetland and grassland habitats in Switzerland (only 2 specimens, collected using window traps) (Prescher et al. 2000). One possibility is that $M$. verralli has soil-dwelling larvae and the emergence trap method could be more efficient and/or that this species is rare this far north in the Palaearctic Region.

The reason for differences in dominant species and species numbers (at least some of them) caught on different localities (i.e., Ticino Forest, pine plantations of the Polish Lowland) may be that different collecting methods are prone to catch different species, which in turn could affect the assemblage structure. For example, the emergence traps collect a group of species that hatch from a small spot on the ground, whereas a Malaise trap is designed to catch species flying around exhibiting positive phototaxis. However, comparisons of different types of trap techniques applied in collecting phorids have rarely been attempted, and those that have been made showed different results and therefore are difficult to assess, see for example Disney et al. (1982), Frouz (1999), Brown \& Feener (1995), Brown (1996), Hancock \& Ward 1996, Weber \& Schiegg (2001). Other aspects affecting species and number of species caught are the number of trap days and collecting periods. i.e., more trap days will catch more species and vice versa, and different collecting periods may cover different weather conditions, i.e., days with good or bad weather.

We found that six of the fourteen dominant species of the megadiverse genus Megaselia (M. pleuralis, M. nigriceps, M. pulicaria-complex, M. brevicostalis, M. unicolor and M. pumila) were also the dominants in the Ticino Forest and in the pine plantations of the Polish Lowland (Durska 2001, 2006, 2009, Prescher et al. 2002). Adding the hurricane of the Pisz Forest in 2002 (Durska 'in prep.'), the dominants of the dis- turbed habitats mentioned above were $M$. pleuralis, M. pulicaria-complex and M. brevicostalis. Thus, we suggest that these three species may be considered to be "phorid disturbance indicators". These species are probably dominants (species of relative abundance $\geq 1 \%$ ) in disturbed areas in general, as they are good colonizers, tolerant to abiotic stress, and generalists with a multivoltine life cycle, which has been discussed before by Durska $(2001,2006,2009)$ and Prescher et al. (2002). Thus, it is possible that these species benefit from habitat heterogeneity followed by various disturbance sources like cutting, wildfires or wind throw, which may create very similar conditions for habitat-insect interactions (Simberloff 1988, Saunders et al. 1991, Tscharntke \& Brandl 2004).

Tyresta Forest was the locality with the highest observed species richness (Table 4; these are only preliminary results due to problems mention before) and estimated species richness compared with the Ticino Forest, the pine plantations (after clear-cutting) of the Białowieża Forest, the Tuchola Forest, the Biała Forest or the Pisz Forest after windthrow in 2002 (Durska 2006, 2009 and 'in prep.', Prescher et al. 2002).

High species richness in wildfire localities during early succession has several explanations. Catastrophic events such as fires and storms create sun-exposed coarse woody debris (CWD). CWD serves as microhabitats for many plants and animals species. In turn, the clearcut harvesting removes most of the stand's biomass (Bouget \& Duelli 2004, Price et al. 1998).

Species associated with disturbed areas have good dispersal power and may colonize a burnt area from the surrounding patches (Southwood 1962). Some flies are early colonizers attracted to the burnt habitat by smoke and/or heat. However, only a few studies have dealt with assemblages of Diptera in wildfire sites, except for some studies of the phenomenon of some species building up swarms in smoke. This is known for species of Microsania (Platypezidae) from nemoral forests in the Palaearctic Region (Belgium, England) (Chandler 1978). Hormopeza (Empididae) has been reported to hunt for Microsania in burnt areas. On the major fire-site from 1999 in the Tyresta Forest, two smoke-attraction experiments were conducted during the middle of the sum- 
mers of 2000 and 2002. These experiments showed that swarms of Microsania also occurred in hemiboreal forests in the Palearctic Region and produced catches of Microsania and Hormopeza (Ahnlund et al. 2006). No scuttle flies were caught during the experiments. However, such experiments should in the future be conducted also in the spring and autumn, during the peak activity periods of scuttle flies.

The effect of fire on the humus layer within the large wildfire site was strong in many places, leaving only the mineral layer of the soil, making evaporation strong. Shelter created by the root systems of the many toppled-over trees, especially old Pinus, might have created a microclimate more suitable for scuttle flies than expected, especially compared with the much more common scenario of moderately burned sites where most trees are standing for a long time. Even though phorids were numerous, Sciaridae were more than twice as abundant (Menzel \& Viklund 'in prep.') and these lesser fungus gnats might have developed in the mycelia in the soil and in the fruiting bodies of the pioneering fungi (Ascomycetes). Consequently, it is possible that many species of scuttle flies are predators/parasitoids on the very abundant Sciaridae. For example, $M$. obscuripennis caught in the survey is a parasitoid on Sciaridae (Disney 1994).

The autumn rise and fall in abundances and richness of Megaselia species is probably connected with the abundance of fungi, directly or via fungi dependent prey species/hosts (Disney 1994, Durska 1996, 2001, 2006, 2009).

Fire and other disturbances, such as clear-cutting of mature stands or wind throw initiate secondary succession of the ecosystem and are "the main source" of habitat heterogeneity. It probably affects diversity and abundance of Phoridae. Many scuttle fly species are known to be very sensitive to changes in their habitat, especially to climatic conditions (Folgarait et al. 2007). During dry years, $M$. brevicostalis reaches the abundance peak much earlier than in years when humidity is typical (Disney et al. 1981). The openarea species of scuttle fly assemblages respond in a parallel way to different disturbances. The same response to disturbances (clear-cutting, grazing and burning) has also been found for spider and carabid fauna (Coddington et al. 1996, Gibson et al. 1992, Zulka et al. 1997, Moretti et al. 2002, Fernández \& Salgado Costas 2004, Huber \& Baumgarten 2005).

\section{Conclusions}

Our investigation showed that there was high species diversity and abundance of scuttle flies after the wildfires. Our results suggest that the scuttle fly assemblages react in a resilient way to environmental disturbance/stress and that Phoridae could be a prime candidate for use in conservation evaluation exercises, in spite of the imperfect knowledge of the alpha taxonomy of this family (Manson et al. 2007, Disney \& Durska 2008). It is in turn possible for Phoridae - as a resilient group of very high ecological diversity - to be used in the monitoring of changes in terrestrial habitats following disturbances, or else as indicators within different ecological levels of organization (Durska 2009). Further studies of this are needed.

Acknowledgements. The majority of the material was collected during a post-fire documentation program funded and initiated by the Swedish Environmental Protection Agency and the Tyresta Forest Foundation. Ewa Durska has benefited from SYNTHESYS support made available by the European Community - Research Infrastructure Action under the FP6 Structuring the European Area Programme AT-TAF 543 and SE-TAF 1833. James Bonet has funding from the Swedish Species Information Centre and the research is a part of the "Swedish Taxonomy Initiative". For critical review of the manuscript and valuable suggestions the authors wishes to thank two anonymous referees for their helpful comments. We also thank Sven Olof Ulefors for determination of scuttle fly species; Thomas Pape, Lars-Ove Wikars and Mark Wolfe for valuable comments on the manuscript.

\section{References}

Ahnlund, H., Viklund, B. \& Wikars, L.-O. 2006: Insekterna. Branden i Tyresta 1999. Dokumentation av effekterna. - Rapport 5604, Naturvírdsverket.s: 81-107.

Bouget, Ch. \& Duelli, P. 2004: The effects of windthrow on forest insect communities: a literature review. Biological Conservation 118: 281-299.

Brown, B. V. 1996. A. Phorid collecting activities in Ecuador, 1996: B. Complementary collecting techniques. - Phorid Newsletter 5: 1-4.

Brown, B. V. \& Feener, Jr. D. H. 1995: Efficiency of two 
mass sampling methods for sampling phorid flies (Diptera: Phoridae) in a tropical biodiversity survey. - Contributions in Science 459: 1-8.

Chandler, P. J. 1978: Some dipterous opportunists at Windsor Forest, Berks.: The attractions for flies of bonfires, wood ash and freshly cut logs - Entomologist's Gazette 29: 253-257.

Coddington, J. A., Young, L. H. \& Coyle, F. A. 1996: Estimating spider species richness in a southern Appalachian cove hardwood forest. - Journal of Arachnology 24: 111-128.

Chao, A. 1984: Non-parametric estimation of the number of classes in a population. - Scandinavian Journal of Statistics 11: 265-270.

Chao, A. 1987: Estimating the population size for capturerecapture data with unequal catchability. - Biometrics 43: 783-791.

Chao, A. \& Shen, T-J. 2003: Program SPADE (Species Prediction And Diversity Estimation). Version 3.1. Available from URL http://chao.stat.nthu.edu.tw. (Accessed 5 March, 2007)

Colwell, R. K. \& Coddington, J. A. 1994: Estimating terrestrial biodiversity through extrapolation. - Philosophical Transactions of the Royal Society of London. Series B, Biological Sciences 345: 101-118.

Danks, H. V. \& Foottit, R. G. 1989: Insects of the boreal zone of Canada. - Canadian Entomologist 121: 625690.

DeBano, L. F., Neary, D. G. \& Ffolliott, P. F. 1998: Fire' s Effects on Ecosystems. - John Wiley \& Sons, New York. 333 pp.

Disney, R. H. L. 1994: Scuttle flies: The Phoridae. Chapman \& Hall, London. 467 pp.

Disney, R. H. L. 2004: Species preferences for white versus yellow water trap for scuttle flies (Dipt., Phoridae). — Entomologist's Monthly Magazine 140: 31-35.

Disney, R. H. L., Coluson, J. C. \& Butterfield, J. 1981: A survey of the scuttle flies (Diptera: Phoridae) of upland habitats in Northern England. - Naturalist, Hull 106: 53-66.

Disney, R. H. L., Erzinclioglu, Y. Z., Henshaw, D. J. de C., Howse, D., Unwin, D. M., Withers, P. \& Woods, A. 1982: Collecting methods and the adequacy of attempted fauna surveys, with reference to the Diptera. - Field Studies 5: 607-621.

Disney, R. H. L. \& Durska, E. 2008: Conservation evaluation and the choice of faunal taxa to sample. - Biodiversity and Conservation 17: 449-451.

Durska, E. 1996: The species composition and structure of scuttle fly communities (Diptera: Phoridae) in mature tree stands in pine forests at different stages of habitat degradation. - Fragmenta Faunistica 39: 267-285.

Durska, E. 2001: Secondary succession of scuttle fly (Diptera: Phoridae) communities in moist pine forest in the Białowieża Forest. — Fragmenta Faunistica 44: 81130.

Durska, E. 2006: Diversity of the scuttle fly (Diptera: Phoridae) communities in the plantations of moist pine forests of the Białowieża Primeval Forest and the Tuchola Forest (Poland). — Biodiversity and Conservation
15: $385-393$

Durska, E. 2009: The scuttle fly (Diptera: Phoridae) assemblages of pine plantations of the Biała Forest (Poland). - Entomologica Fennica 20: 170-178.

Fernández, M. M. \& Salgado Costas, J. M. 2004: Recolonization of burnt pine forest (Pinus pinaster) by Carabidae (Coleoptera). — European Journal of Soil Bio$\operatorname{logy}$ 40: 47-53.

Folgarait, P. J., Patrock, R. J. W. \& Gilbert, L. E. 2007: The influence of ambient conditions and space on the phenological patterns of a Solenopsis phorid guild in an arid environment. - Biological Control 42: 262-273.

Frouz, J. 1999: Adult migration of soil dwelling Diptera with respect to vegetation cover, succession age and larval breeding sites - preliminary results. - In: Tajovsky K., Pizl V. (eds.), Soil Zoology in Centyral Europe. Ceské Budejovice: ISB AS CR: 53-62.

Gibson, C. W. D., Hambler, C. \& Brown, V. K. 1992: Changes in spider (Araneae) assemblages in relation to succession and grazing management. - Journal of Applied Ecology 29: 132-142.

Hancock, E. G. \& Ward, A. 1996: The effect of shade on the relative abundance of insects in water traps in the tropics. — Entomologist 115: 91-96.

Hirowatari, T., Makihara, H. \& Sugiarto 2007: Effects of fires on butterfly assemblages in lowland dipterocarp forest In East Kalimantan. - Entomological Science 10: $113-127$.

Holliday, N. J. 1991: Species responses of carabid beetles (Coleoptera: Carabidae) during post-fire regeneration of boreal forest. - Canadian Entomologist 123: 1369-1389.

Huber, Ch. \& Baumgarten, M. 2005: Early effects of forest regeneration with selective and small scale clear-cutting on ground beetles (Coleoptera, Carabidae) in a Norway spruce stand in Southern Bavaria (Höglwald). - Biodiversity and Conservation 14: 1989-2007.

Koivula, M., Cobb, T., Déchęne, A. D., Jacobs, J. \& Spence, J. R. 2006: Responses to two Sericoda Kirby, 1837 (Coleoptera: Carabidae) species to forest harvesting, wildfire and burn severity. - Entomologica Fennica 17: $315-324$.

Manson, N. W. H., Wilson, J. B. \& Steel, J. B. 2007: Are alternative stable states more likely in high stress environments? Logic and available evidence do not support Didham et al. 2005. — Oikos 116: 353-357

Moretti, M., Conedera, M., Duelli, P. \& Edwards, P. J. 2002: The effects of wildfire on ground-active spiders in deciduous forests on the Swiss southern slope of the Alps. - Journal of Applied Ecology 39: 321-336.

Muona, J. \& Rutanen, I. 1994: The short-term impact of fire on the beetle fauna in boreal coniferous forest. Annales Zoologici Fennici 31: 109-121.

Niklasson, M. \& Granström, A. 2000: Numbers and size of fires: long-term spatially explicit fire history in a Swedish boreal landscape. - Ecology 81: 1484-1499.

Petterson, U. 2006: Introduktion. Branden i Tyresta 1999. Dokumentation av effekterna. — Rapport 5604, Naturvírdsverket.

Prescher, S., Obrist, M. K. \& Duelli, P. 2000: Die Phori- 
denfauna (Diptera, Brachycera) naturnaher Biotope und intensiv genutzter Kulturflächen im Schweizer Mitteland. - Mitteilungen der Schweizerischen Entomologischen Gesellschaft. Bulletin de la Société Entomologique Suisse 73: 265-275.

Prescher, S., Moretti, M. \& Duelli, P. 2002: Scuttle flies (Diptera, Phoridae) in Castanea sativa forests in the southern Alps (Ticino, Switzerland), with thirteen species new to Switzerland. - Mitteilungen der Schweizerischen Entomologischen Gesellschaft. Bulletin de la Société Entomologique Suisse 75: 289-298.

Price, K., Pojar, J., Roburn, A., Brewer, L. \& Poirier, N. 1998: Windthrown or clearcur - what's the difference? - Northwest Science 72: 30-33.

Saunders, D., Hobbs, R. J. \& Margules, C. R. 1991: Biological consequences of ecosystem fragmentation - a review. - Conservation Biology 5: 18-32.

Scharff, N., Coddington, J. A., Griswold, C. E., Hormiga, G. \& Bjřrn, P. D. 2003: When to quit? Estimating spider species richness in a northern European deciduous forest. - Journal of Arachnology 31: 246-273.

Simberloff, D. 1988: The contribution of population and community biology to conservation science. - Annual Review of Ecology and Systematics 19: 473-51.

Southwood, T. R. E. 1962: Migration of terrestrial arthropods in relation to habitat. - Biological Review 37: $171-214$

Swengel, A. B. 2001: A literature review of insect respon- ses to fire, compared to other conservation managements of open habitat. - Biodiversity and Conservation 10: 1141-1169.

Toivanen, T. \& Kotiaho, J. S. 2007: Mimicking natural disturbances of boreal forests: the effects of controlled burning and creating dead wood on beetle diversity. Biodiversity and Conservation 16: 3193-3211.

Tscharntke, T. \& Brandl, R. 2004: Plant-insect interactions in fragmented landscapes. - Annual Review of Entomology 49: 405-430.

Walther, B. A. \& Martin, J.-L. 2001: Species richness estimation of bird communities: how to control for sampling effort? - Ibis 143: 413-419.

Weber, G. \& Schiegg, K. 2001: Scuttle flies (Diptera: Phoridae) from the forest reserve Sihlwald $\mathrm{ZH}$. — Studia Dipterologica 8: 271-276.

Wikars, L.-O. \& Schimmel, J. 2001: Immediate effects of fire-severity on soil invertebrates in cut and uncut pine forests. - Forest Ecology and Management 141: 189 200.

Zackrisson, O. 1977: Influence of forest fires on the North Swedish boreal forest. - Oikos 29: 22-32.

Zulka, K. P., Milasowszky, N. \& Lethmayer, Ch. 1997: Spider biodiversity potential of an ungrazed and grazed inland salt meadow in the National Park ,Neusiedler See-Seewinkel" (Austria): implications for management (Arachnida: Araneae). - Biodiversity and Conservation 6: 75-88. 